\title{
Variabilite Des Extremes Pluviometriques Sur Le Bassin Versant De La Riviere Bia (Sud-Est, Cote d'Ivoire)
}

\section{Kouassi Kan Martin}

Doctorant à l'UFR Sciences de la Terre et des Ressources Minières Université Félix Houphouët Boigny Abidjan Cocody, Côte d'Ivoire

\section{Kouassi Kouakou Lazare}

Maitre de Conférence, Directeur de l'UFR-Environnement Université Jean Lorougnon Guede Daloa, Côte d'Ivoire

\section{Yao Koffi Blaise}

Maitre de Conférence

à l'UFR- Science de la Terre et des Ressources Minières

Université Félix Houphouët Boigny Abidjan Cocody, Côte d'Ivoire

\section{Meledje N'diaye Hermann}

Assistant, Attaché de Recherche au Centre de Recherche en Ecologie

Université Nangui Abrogoua Abidjan, Côte d'Ivoire

\section{Biemi Jean}

Professeur Titulaire, Doyen Honoraire

de l'UFR-Science de la Terre et des Ressources Minières, Côte d'Ivoire

\section{Lasm Théophile}

Maître de conférences l'UFR- Science de la Terre et des Ressources Minières, Responsable du Laboratoire Hydrogéologie Université Félix Houphouët Boigny Abidjan Cocody, Côte d'Ivoire

\section{Nathalie Rouché}

Professeur Titulaire, Gestionnaire de Base de Données à l'Institut de Recherche et de DéveloppementHydrosciences Montpellier, France

Doi: 10.19044/esj.2018.v14n2p134 URL:http://dx.doi.org/10.19044/esj.2018.v14n2p134

\begin{abstract}
Climate change is a reality which affects many climatic variables, including precipitation. The objective of this article is to study the extreme rain variability from a historical database (1941 - 2000). For this purpose, eleven (11) rainfall indices were calculated at the Adiaké, Bianouan, Ayamé, and Agnibilekro rainfall stations in Côte d'Ivoire. Due to lack of data on daily rainfall on the Ghanaian section of watershed, nine (9) monthly precipitations indices were estimated from monthly rainfall at the Enshi and Buakuc stations.
\end{abstract}


The trend analysis of the precipitations indices show that a generalized downward was most significant in Adiaké compared to other stations. Furthermore, indices extreme thresholding (P99, P99p, P99,5 and P99,5p) have experienced a stable trend and a stationary evolution. All steps were detected in the non-stationary indices mostly observed after 1980 (late break) and before 1960 (precocious break).

Keywords: Rainfall indices, Extreme rainfall, Bia watershed

\section{Résumé}

L'impact de la variabilité du climat sur la pluie extrême peut s'avérer plus dommageable qu'une simple variation de la moyenne pluviométrique. L'objectif poursuivi par cet article est donc d'étudier la variabilité de la pluie extrême sur le bassin versant de la Bia à partir d'une longue base de données (1941 - 2000). Pour ce faire, onze (11) indices de pluie journalière ont été calculés aux stations pluviométriques d'Adiaké, de Bianouan, d'Ayamé et d'Agnibilékro en Côte d'Ivoire. Sur la partie ghanéenne du bassin, neuf (9) indices de pluie mensuelle ont été calculés à partir de la pluie mensuelle issue des stations de Enshi et de Buakuc. L'analyse de la tendance des indices calculés révèle une baisse généralisée qui a été plus significative à Adiaké et à Enshi. Par ailleurs, les indices de seuillages (P99, P99p, P99,5 et P99,5p) ont connu une tendance stable et une évolution stationnaire. Cependant, toutes les ruptures détectées dans les indices non stationnaires s'observent majoritairement après 1980 (ruptures tardives) et avant 1960 (ruptures précoces).

Mots-clés: Indices pluviométriques, précipitations extrêmes, bassin versant de la Bia

\section{Introduction}

La réalité d'une variabilité climatique fait désormais l'objet d'un consensus bien affirmé (Ouédraogo et al., 1998; Kouassi et al., 2010). Aussi, sa répercussion sur la pluviométrie moyenne constitue une réalité en Afrique (Ouédraogo et al., 1998; Servat et al., 1998; Conway et al., 2009) et en Côte d'Ivoire (Paturel et al., 1998; Bigot et al., 2002; Kouassi et al., 2010) depuis la fin des années 1960 et le début des années 1970. Cependant, l'impact de la variabilité du climat sur la pluie extrême a été très peu étudié (Assani, 1999; Goula et al., 2012). L'une des principales raisons évoquée est la difficulté d'accès aux données qualitatives (pluie journalière, débit minimal ou maximal...). Pourtant une variation de la pluie extrême peut s'avérer plus dommageable que celle de la moyenne (Lemaître, 2002). 
L'objectif de la présente étude est donc de combler ce déficit. Elle se propose ainsi d'étudier la variabilité de la pluie extrême sur le bassin versant de la Bia en utilisant les indices de pluie. En effet, ces indices sont des variables qualitatives permettant de caractériser les variables climatiques (la pluie, le débit, la température...) en leurs parties extrêmes. Elles sont recommandées par la communauté scientifique pour étudier la répercussion du climat sur les variables extrêmes (Christensen et al., 2002). En Afrique de l'ouest, l'utilisation des indices de température, de pluies et de débit a permis d'apprécier la variabilité interannuelle de ces variables dans leurs parties extrêmes (Ozer et al., 2014; Karimou et al., 2015; Hangnon et al., 2015). Sur le bassin versant de la Bia, nous testons l'utilisation des indices de pluie de 1941 à 2000.

\section{Présentation de la Zone D'étude}

La Bia est une rivière transfrontalière entre la Côte d'Ivoire et le Ghana ; elle coule entre $5^{\circ} 30^{\prime}$ et $5^{\circ} 50^{\prime}$ de latitude Nord et entre $3^{\circ}$ et $3^{\circ} 15^{\prime}$ de longitude Ouest (Figure 1).

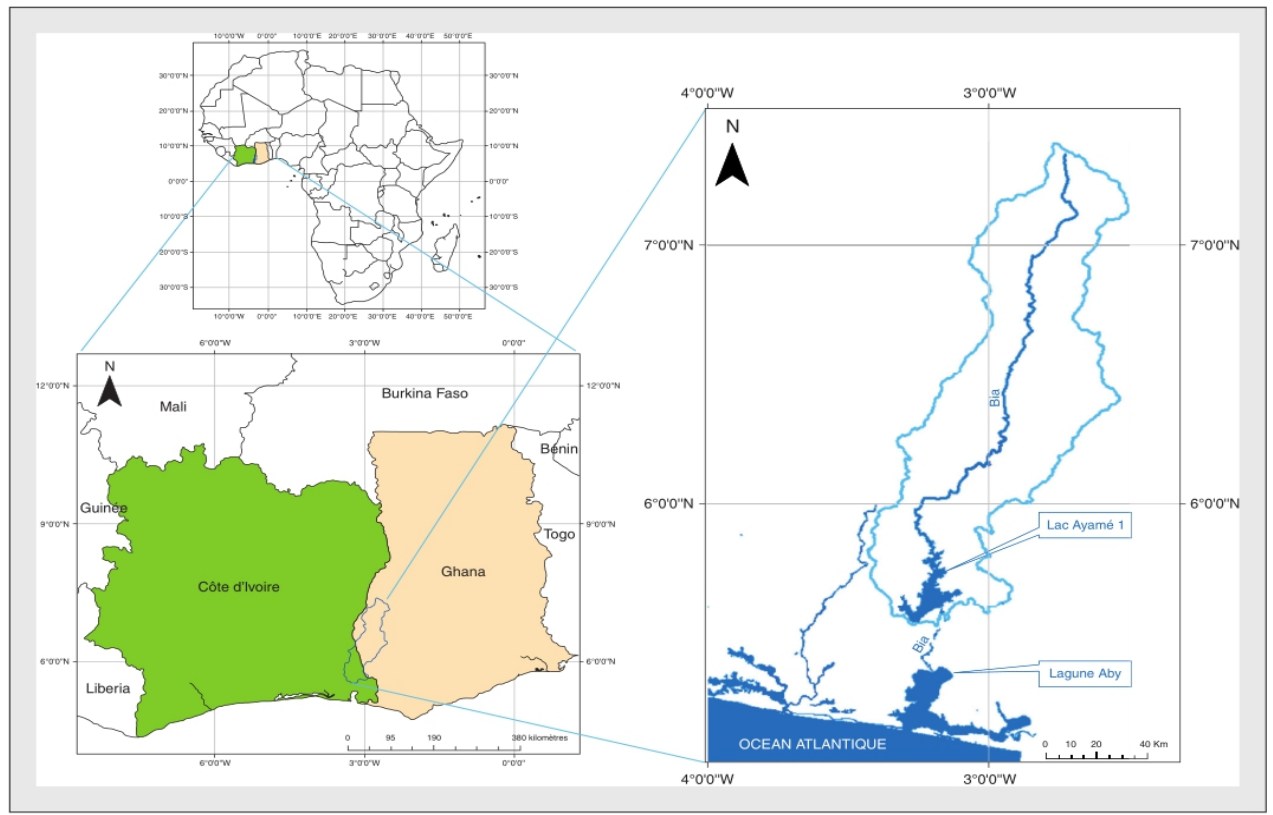

Figure 1. Localisation de la zone d'étude (Meledje et al., 2015)

Elle prend sa source à Kérimasso au nord du Ghana et se déverse dans la lagune Aby au sud de la Côte d'Ivoire (Reizer, 1967). De forme allongée, le bassin versant de la Bia couvre une superficie de $9650 \mathrm{~km}^{2}$ dont $2 / 3$ sont situées en territoire ghanéen. L'analyse pluviométrique faite par Meledje (2016), permet de distinguer deux (2) zones climatiques sur le bassin le bassin de la Bia. Une zone tropicale humide $(1460 \mathrm{~mm})$ correspondant au nord du 
bassin avec une seule saison des pluies et une zone subéquatoriale (1870 $\mathrm{mm})$ correspond au sud du bassin avec deux saisons des pluies distinctes.

\section{Présentation des Données}

Pour cette étude, nous disposons de données journalières fournies par l'IRD (Institut de Recherche et de Développement) du $1^{\text {er }}$ janvier 1941 au 31 décembre 2000 aux stations d'Ayamé, d'Adiaké, d'Agnibilékro et de Bianouan couvrant la zone de la Côte d'Ivoire (Boyer et al., 2006). Au niveau du Ghana par faute de données journalières, les données mensuelles fournies par la base de données IDIS (Integrated Database Information System) de janvier 1941 à décembre 2000 aux stations d'Enshi et Buakuc ont été utilisées. Les caractéristiques des données issues des différentes stations sont présentées dans le Tableau 1.

\begin{tabular}{ccccccc}
\hline Stations & $\begin{array}{c}\text { Latitude } \\
(\mathrm{DD})\end{array}$ & $\begin{array}{c}\text { Longitude } \\
(\mathrm{DD})\end{array}$ & $\begin{array}{c}\text { Pluviométrie } \\
\text { moyenne } \\
(\mathrm{mm})\end{array}$ & $\begin{array}{c}95^{\mathrm{e}} \\
\text { centile } \\
(\mathrm{mm})\end{array}$ & $\begin{array}{c}99^{\mathrm{e}} \text { centile } \\
(\mathrm{mm})\end{array}$ & $\begin{array}{c}99,5^{\mathrm{e}} \\
\text { centile } \\
(\mathrm{mm})\end{array}$ \\
\hline Adiaké & 5,3 & $-3,3$ & 5,3 & 31,90 & 77,19 & 96,74 \\
\hline Agnibilékro & 7,12 & $-3,2$ & 3,2 & 22,50 & 48,20 & 60,54 \\
\hline Ayamé & 5,6 & $-3,17$ & 4,1 & 23,30 & 55,22 & 71,76 \\
\hline Bianouan & 6,03 & $-3,2$ & 4,0 & 20,30 & 55,62 & 78,77 \\
\hline Enshi & 5,82 & $-2,82$ & 126,18 & 276 & 346,81 & 362 \\
\hline Buakuc & 6,4 & $-2,58$ & 118,07 & 315 & 415,24 & 444,215 \\
\hline
\end{tabular}

Tableau 1. Caractéristiques des six (6) stations pluviométriques (les valeurs de centile sont calculées sur la période 1956-1985)

\section{Méthodologie}

\section{* Calcul des Indices de Pluie}

Les indices de pluies ont été calculés à l'échelle journalière aux stations d'Adiaké, d'Ayamé, de Bianouan et d'Agnibilékro en Côte d'Ivoire à partir de la pluie journalière. Cependant, par faute de données de pluie à l'échelle journalière dans la partie ghanéenne du bassin, les indices de pluie ont été calculés à l'échelle mensuelle sur cette zone du bassin aux stations de Buakuc et de Enshi à partir de la pluie mensuelle.

Les indices de pluies journalières utilisés dans cette étude sont définis par le groupe d'experts sur la détection et les indices du changement climatique (ETCCDI) (http: //etccdi.pacificclimate.org/). Ils reposent généralement sur l'approche du seuillage par centile. Pour l'analyse de la variabilité de la pluie extrême sur le bassin versant de la Bia, onze (11) indices de pluies journalières ont été calculés pour chaque station (Tableau 2). Les centiles sont calculés à partir des données journalières sur une période de trente (30) ans (1956-1985). Cette exigence permet de se conformer aux normes de l'Organisation Météorologique Mondiale (OMM). 


\begin{tabular}{ccc}
\hline Identification & Noms de l'indice & Définition \\
\hline PTOT $(\mathrm{mm})$ & Total pluviométrique & Précipitations totales annuelles \\
\hline JP (jour) & Jour de pluie & $\begin{array}{c}\text { Le nombre total de jours humides (précipitation } \\
\geq 1 \mathrm{~mm})\end{array}$ \\
\hline SDII $(\mathrm{mm} / \mathrm{j})$ & $\begin{array}{c}\text { Indice d'intensité de jours } \\
\text { pluvieux }\end{array}$ & $\begin{array}{c}\text { La lame d'eau moyenne précipitée par jour de } \\
\text { pluie }\end{array}$ \\
\hline PXJA (mm) & $\begin{array}{c}\text { Pluie maximale journalière } \\
\text { PXJAp (\%) }\end{array}$ & $\begin{array}{c}\text { Précipitation maximale quotidienne de chaque } \\
\text { année }\end{array}$ \\
\hline P95 (jour) & $\begin{array}{c}\text { Fréquence de pluie intense } \\
\text { quotidienne }\end{array}$ & $\begin{array}{c}\text { Nombre de jour avec des précipitations } \geq 95^{\mathrm{e}} \\
\text { centile }\end{array}$ \\
\hline P95p (\%) & $\begin{array}{c}\text { La part de la pluviométrie } \\
\text { intense }\end{array}$ & $\begin{array}{c}\text { Proportion des précipitations intenses dans le } \\
\text { total pluviométrique annuel }\end{array}$ \\
\hline P99 (jour) & $\begin{array}{c}\text { Fréquence de pluie extrême } \\
\text { quotidienne dans le total pluviométrique annuel }\end{array}$ & $\begin{array}{c}\text { Nombre de jour avec des précipitations } \geq 99^{\mathrm{e}} \\
\text { centile }\end{array}$ \\
\hline P99p (\%) & $\begin{array}{c}\text { La part de la pluviométrie } \\
\text { extrême }\end{array}$ & $\begin{array}{c}\text { Proportion des précipitations extrêmes dans le } \\
\text { total pluviométrique annuel }\end{array}$ \\
\hline P99,5 (jour) & $\begin{array}{c}\text { Fréquence de pluie très } \\
\text { extrême }\end{array}$ & $\begin{array}{c}\text { Nombre de jour avec des précipitations } \geq 99^{\mathrm{e}} \\
\text { centile }\end{array}$ \\
\hline P99,5p (\%) & $\begin{array}{c}\text { La part de la pluviométrie } \\
\text { très extrême }\end{array}$ & $\begin{array}{c}\text { Proportion des précipitations très extrêmes dans } \\
\text { le total pluviométrique annuel }\end{array}$ \\
\hline
\end{tabular}

Tableau 2. Indices de pluie journalière utilisés dans l'analyse des événements climatiques extrêmes

Sur la partie ghanéenne du bassin de la Bia, neuf (9) indices de pluie mensuelle ont été calculés aux stations de Enshi et Buakuc (Tableau 3). Les six (6) derniers indices du Tableau 3 sont estimés à partir d'une classification de la pluviométrie mensuelle en mois de faible pluviométrie, de pluviométrie intermédiaire et de forte pluviométrie à partir des seuils $100 \mathrm{~mm}$ et $200 \mathrm{~mm}$ de pluie (Bierot, 1965; Brou \& Dieulin, 2008).

\begin{tabular}{ccc}
\hline Identification & Noms de l'indice & Définition \\
\hline PA (mm) & Total pluviométrique & Cumul de la pluie de chaque année \\
\hline PXMA (mm) & Pluie maximale mensuelle & $\begin{array}{c}\text { Précipitation maximale mensuelle de chaque } \\
\text { année }\end{array}$ \\
\hline PXMAp (\%) & $\begin{array}{c}\text { Part de la pluie maximale } \\
\text { mensuelle }\end{array}$ & $\begin{array}{c}\text { Proportion de la précipitation maximale } \\
\text { mensuelle dans le total pluviométrique annuel }\end{array}$ \\
\hline PFa (mois) & Mois de faible pluviométrie & Précipitation $<100 \mathrm{~mm}$ \\
\hline PI (mois) & $\begin{array}{c}\text { Mois de pluviométrie } \\
\text { intermédiaire }\end{array}$ & $100 \mathrm{~mm} \leq$ précipitation $<200 \mathrm{~mm}$ \\
\hline PFo (mois) & Mois de forte pluviométrie & Précipitation $\geq 200 \mathrm{~mm}$ \\
\hline PFap (\%) & $\begin{array}{c}\text { Part de la pluie des mois de } \\
\text { faible pluviométrie }\end{array}$ & $\begin{array}{c}\text { Proportion de la pluie du mois de faible } \\
\text { pluviométrie dans le cumul annuel. }\end{array}$ \\
\hline PIp (\%) & $\begin{array}{c}\text { Part de la pluie des mois de } \\
\text { faible pluviométrie }\end{array}$ & $\begin{array}{c}\text { Proportion de la pluie du mois de } \\
\text { pluviométrie intermédiaire dans le cumul } \\
\text { annuel. }\end{array}$ \\
\hline PFop (\%) & $\begin{array}{c}\text { Part de la pluie des mois de } \\
\text { forte pluviométrie }\end{array}$ & $\begin{array}{c}\text { Proportion de la pluie du mois de forte } \\
\text { pluviométrie dans le cumul annuel. }\end{array}$ \\
\hline
\end{tabular}

Tableau 3. Indices de pluie mensuelle utilisés dans l'analyse des événements climatiques extrêmes 


\section{Analyse de la Variabilité de la Pluie Extrême}

Une analyse de la tendance et de la stationnarité des indices de pluie journalière et mensuelle a permis d'apprécier la variabilité de la pluviométrie extrême sur le bassin de la Bia de 1941 à 2000.

\section{- Analyse des Tendances}

L'analyse des tendances linéaires de la pluie extrême a été réalisée par régression linéaire entre les différents indices de pluie (journalière et mensuelle) et le temps (en années). Les pentes ainsi estimées ont été regroupées en classes indiquant des tendances significatives à la hausse ou à la baisse. La limite des classes considérées est définie à partir de la statistique t de Student utilisée pour tester l'hypothèse d'une pente égale à 0 . La tendance est qualifiée en fonction de la probabilité $p$ du test $t$ appliquée à la pente de régression.

Si $p<0,01$ : la tendance est qualifiée de très significative Si $0,01 \leq p<0,05$ : la tendance est qualifiée de significative Si $p \geq 0,05$ : la tendance est qualifiée de non significative

\section{- Analyse de la Stationnarité de la Pluie Extrême}

La détection de ruptures uniques et multiples dans les indices de pluies journalière et mensuelle a permis d'apprécier la stationnarité de la pluie extrême. Le test de Pettitt (Pettitt, 1979) a été utilisé pour détecter les ruptures uniques tandis que la recherche des ruptures multiples s'est faite avec la procédure de segmentation de Hubert.

L'applicabilité de ces deux méthodes a été vérifiée avec beaucoup de succès dans de nombreuses études en Afrique de l'ouest (Paturel et al., 1998; Lubès-Niel et al., 1998).

\section{Résultats}

\section{* Indices de Pluie Extrêmes}

Le Tableau 4 présente pour chaque station la moyenne des indices de pluie journalière calculée de 1941 à 2000. Ces résultats révèlent une faible répartition des indices moyens à Agnibilékro dans le nord du bassin alors que cette répartition est plus prépondérante au sud avec une forte moyenne des indices PTOT et PXJA à Adiaké et de 1'indice JP à Ayamé. Par ailleurs, la part annuelle de pluie maximale (PXJAp) inférieure à 10\% varie très peu d'une station à une autre. La fréquence des jours de pluie intense (P95) est en moyenne inférieure à 20 jours et contribue à environ $50 \%$ de la pluie annuelle à la majorité des stations. Le nombre de jour de pluie extrême (P99) est d'environ 3 jours et représente moins de $20 \%$ de pluie annuelle à la plupart des stations. Une fréquence moyenne de 1 jour de pluie très extrême $(\mathrm{P} 99,5)$ contribue à moins de $15 \%$ de pluie annuelle. 


\begin{tabular}{ccccc}
\hline $\begin{array}{c}\text { Indices de } \\
\text { pluie }\end{array}$ & Adiaké & Agnibilékro & Ayamé & Bianouan \\
\hline $\begin{array}{c}\text { PTOT } \\
(\mathrm{mm})\end{array}$ & 1937,92 & 1151,51 & 1476,77 & 1462,38 \\
\hline $\begin{array}{c}\text { PXJA } \\
(\mathrm{mm})\end{array}$ & 140,78 & 77,52 & 102,64 & 128,21 \\
\hline PXJAp (\%) & 7,33 & 6,74 & 6,99 & 8,73 \\
\hline JP (jour) & 117,13 & 82,13 & 125,92 & 114,9 \\
\hline P95 (jour) & 17,32 & 15,98 & 16,90 & 18,22 \\
\hline P95p (\%) & 52,34 & 50,54 & 48,99 & 54,30 \\
\hline P99 (jour) & 3,37 & 3,37 & 3,50 & 3,78 \\
\hline P99p (\%) & 18,25 & 17,01 & 18,31 & 22,47 \\
\hline $\begin{array}{c}\text { P99,5 } \\
\text { (jour) }\end{array}$ & 1,95 & 1,62 & 1,78 & 1,9 \\
\hline P99,5p (\%) & 12,32 & 9,62 & 11,16 & 14,42 \\
\hline
\end{tabular}

Tableau 4. Moyenne des indices de pluie journalière

Les moyennes des indices de pluie mensuelle issue des stations de Buakuc et de Enshi en zone ghanéenne sont consignées dans le Tableau 5. L'analyse de ce tableau révèle que la pluie annuelle (PA), la pluie maximale mensuelle (PXMA) ainsi que sa part de pluie (PXMAp) sont en moyennes plus élevées à Enshi qu'à Buakuc.

\begin{tabular}{cccccccccc}
\hline $\begin{array}{c}\text { Indice } \\
\text { de pluie }\end{array}$ & $\begin{array}{c}\text { PA } \\
(\mathrm{mm})\end{array}$ & $\begin{array}{c}\text { PXMA } \\
(\mathrm{mm})\end{array}$ & $\begin{array}{c}\text { PXMAp } \\
(\%)\end{array}$ & $\begin{array}{c}\text { PFa } \\
(\text { mois })\end{array}$ & $\begin{array}{c}\text { Pfap } \\
(\%)\end{array}$ & $\begin{array}{c}\text { PI } \\
(\text { mois })\end{array}$ & $\begin{array}{c}\text { Pip } \\
(\%)\end{array}$ & $\begin{array}{c}\text { Pfo } \\
(\text { mois })\end{array}$ & $\begin{array}{c}\text { Pfop } \\
(\%)\end{array}$ \\
\hline Buakuc & 1416,83 & 283,05 & 20,18 & 5,52 & 17,78 & 4,22 & 42,79 & 2,27 & 39,43 \\
\hline Enshi & 1514,15 & 332,40 & 22,09 & 5,73 & 18,88 & 3,80 & 36,55 & 2,47 & 44,57 \\
\hline
\end{tabular}

Tableau 5. Moyenne des indices de pluie mensuelle

Par ailleurs pour les deux (2) stations, les mois de faible pluviométrie ( $\mathrm{PFa})$ plus prépondérants avec une moyenne presque constante (5 mois), contribuent à la plus faible proportion de pluie annuelle (moins de 20\%). Cependant, à Enshi les mois de forte pluie ( $\mathrm{PFo}$ ) sont en moyenne les plus faibles $(2,47$ mois) mais représentent la plus grande proportion $(44,57 \%)$ de pluie annuelle alors qu'à Buakuc ce sont en moyenne 4,22 mois de pluie intermédiaire (PI) qui contribuent à la plus grande proportion $(42,79 \%)$ de pluie annuelle (Tableau 5).

\section{* Tendances de la Pluie Extrême}

La tendance des indices de pluie journalière estimée en pourcentage de décennie est résumée dans le Tableau 6 . Les valeurs négatives indiquent des tendances en baisse et les valeurs positives des tendances en hausse. Les signes $(* *)$ et $(*)$ indiquent respectivement des tendances très significative et significative. L'absence de signe montre une tendance non significative. 


\begin{tabular}{ccccc}
\hline Indices de pluie & Adiaké & Agnibilékro & Ayamé & Bianouan \\
\hline PTOT $(\mathrm{mm})$ & $-0,48^{* *}$ & $-0,45$ & $-0,46^{* *}$ & $-0,45$ \\
\hline PXJA $(\mathrm{mm})$ & $-0,45$ & $-0,43$ & $-0,43$ & $-0,39$ \\
\hline PXJAp $(\%)$ & 0,58 & 0,17 & 0,65 & 0,64 \\
\hline JP $($ jour $)$ & $-0,42^{*}$ & 4,45 & 0,07 & $-0,32$ \\
\hline SDII $(\mathrm{mm} / \mathrm{j})$ & $-0,47^{* *}$ & $-0,45$ & $-0,47^{* *}$ & $-0,44$ \\
\hline P95 (jour) & $-0,48^{* *}$ & $-0,46$ & $-0,48^{* *}$ & $-0,42$ \\
\hline P95p (\%) & $-0,44^{*}$ & $-0,42$ & $-0,43^{*}$ & $-0,07$ \\
\hline P99 (jour) & $-0,48^{* *}$ & $-0,46$ & $-0,48$ & $-0,47$ \\
\hline P99p (\%) & $-0,48^{*}$ & $-0,44$ & $-0,45$ & $-0,45$ \\
\hline P99,5 (jour) & $-0,49^{* *}$ & $-0,48$ & $-0,48$ & $-0,49^{*}$ \\
\hline P99,5p (\%) & $-0,48^{* *}$ & $-0,47$ & $-0,47$ & $-0,48$ \\
\hline
\end{tabular}

Tableau 6. Tendance exprimées en $\%$ de décennie des indices de pluie journalière

À partir de ces indications, l'analyse des résultats du Tableau 6 montre une tendance non significative (stabilité) dans la majorité des indices étudiés à Agnibilékro et à Bianouan. La grande instabilité est observée à Adiaké avec six (6) indices (PTOT, SDII, P95, P99, P99,5 et P99,5) à tendances négatives très significatives et trois (3) autres (JP, P95p et P99p) à tendances négatives significatives. A Ayamé, cette instabilité est caractérisée par une baisse significative de la pluie annuelle (PTOT), de la lame d'eau écoulée (SDII) et de la fréquence de pluie intense (P95).

La tendance des indices de pluie mensuelle en $\%$ de décennie révèle à Buakuc une tendance non significative, qui est négative pour six (6) indices (PA, PXMA, PXMA, PI, PFo et PFo) et positive pour trois (3) indices (PFa, PFa et PIp) (Tableau 7).

\begin{tabular}{cccccccccc}
\hline Stations & PA (mm) & PXMA (mm) & PXMAp (\%) & PFa (mois) & PFap (\%) & PI (mois) & PIp (\%) & PFo (mois) & PFop (\%) \\
\hline Buakuc & $-0,4$ & $-0,4$ & $-0,38$ & 0,62 & 0,62 & $-0,31$ & 0,66 & $-0,46$ & $-0,44$ \\
\hline Enshi & $-0,44^{* *}$ & $-0,44^{* *}$ & 0,61 & $-0,44^{*}$ & $-0,23$ & 1,13 & 0,56 & $-0,48^{* *}$ & $-0,47^{* *}$ \\
\hline
\end{tabular}

Tableau 7. Tendance (\% de décennie) type indice de pluie mensuelle

Cependant à Enshi, la tendance à la baisse est significative pour l'indice PFa et très significative pour les indices PA, PXMA, FPo et FPop tandis que les autres indices (PXMAp, PFap, PI et PIp) connaissent une stabilité.

\section{Stationnarité de la Pluie Extrême}

L'application des tests de Pettitt et de Hubert aux indices de pluie journalière a permis d'apprécier la stationnarité de la pluie extrême entre 1941 et 2000 aux différentes stations. Les résultats sont résumés dans le Tableau 8 où le signe (-) indique l'absence de rupture. 


\begin{tabular}{|c|c|c|c|c|c|c|c|c|}
\hline Stations & \multicolumn{2}{|c|}{ Adiaké } & \multicolumn{2}{|c|}{ Agnibilékro } & \multicolumn{2}{|c|}{ Ayamé } & \multicolumn{2}{|c|}{ Bianouan } \\
\hline Tests statistiques & $\begin{array}{c}\text { Méthode } \\
\text { de } \\
\text { Pettitt }\end{array}$ & $\begin{array}{c}\text { Méthode } \\
\text { de } \\
\text { Hubert }\end{array}$ & $\begin{array}{c}\text { Méthode } \\
\text { de } \\
\text { Pettitt }\end{array}$ & $\begin{array}{c}\text { Méthode } \\
\text { de } \\
\text { Hubert }\end{array}$ & $\begin{array}{c}\text { Méthode } \\
\text { de } \\
\text { Pettitt }\end{array}$ & $\begin{array}{l}\text { Méthode } \\
\text { de } \\
\text { Hubert }\end{array}$ & $\begin{array}{c}\text { Méthode } \\
\text { de } \\
\text { Pettitt }\end{array}$ & $\begin{array}{c}\text { Méthode } \\
\text { de } \\
\text { Hubert }\end{array}$ \\
\hline PTOT (mm) & 1982 & 1982 & - & 1995 & 1980 & 1980 & - & - \\
\hline PXJA (mm) & - & - & - & - & 1979 & - & - & 1981 \\
\hline PXJAp (\%) & - & - & - & - & - & - & - & - \\
\hline JP (jour) & 1982 & 1982 & - & 1993 & - & 1969 & - & - \\
\hline $\mathrm{SDII}(\mathrm{mm} / \mathrm{j})$ & 1983 & - & - & $\begin{array}{c}1954 \text { et } \\
1995\end{array}$ & 1979 & 1979 & - & - \\
\hline P95 (jour) & 1982 & 1982 & - & $\begin{array}{c}1954 \text { et } \\
1995\end{array}$ & 1980 & 1980 & - & - \\
\hline P95p (\%) & 1983 & - & - & $\begin{array}{c}1948 \text { et } \\
1995\end{array}$ & 1983 & 1983 & - & 1953 \\
\hline P99 (jour) & 1983 & 1983 & - & - & - & - & - & - \\
\hline P99p (\%) & - & - & - & 1995 & - & - & - & - \\
\hline P99,5 (jour) & - & - & - & - & - & - & - & - \\
\hline P99,5p (\%) & - & - & - & - & - & - & - & - \\
\hline
\end{tabular}

Tableau 8. Stationnarité des indices de pluie journalière

Cette stationnarité est caractérisée par une absence de rupture multiple dans les indices de pluie journalière à Adiaké et à Ayamé de 1941 à 2000 selon le test de Hubert. Toutefois, cette loi détecte à Agnibilékro une double rupture (1948 et 1995) dans l'indice P95p, puis une autre (1954 et 1995) dans les indices SDII et P95. En outre, le test de Pettitt accepte l'hypothèse d'une rupture dans cinq (5) indices à Ayamé (PTOT, PXJA, SDII, P95 et P95p), puis dans six (6) autres à Adiaké (PTOT, JP, SDII, P95, P95p et P99) alors qu'aux stations de Bianouan et d'Agnibilékro cette hypothèse est rejetée pour l'ensemble des indices étudiés.

Le Tableau 9 permet d'apprécier les dates de ruptures dans la pluie extrême aux stations de Enshi et Buakuc sur la partie du bassin située au Ghana.

\begin{tabular}{|c|c|c|c|c|c|c|c|c|c|c|}
\hline Stations & Tests statistiques & $\begin{array}{c}\text { PA } \\
(\mathrm{mm})\end{array}$ & $\begin{array}{c}\text { PXMA } \\
(\mathrm{mm})\end{array}$ & $\begin{array}{c}\text { PXMAp } \\
(\%)\end{array}$ & $\begin{array}{c}\mathrm{PFa} \\
\text { (mois) }\end{array}$ & $\begin{array}{c}\text { PFap } \\
(\%)\end{array}$ & $\begin{array}{c}\text { PI } \\
\text { (mois) }\end{array}$ & $\begin{array}{l}\text { PIp } \\
(\%)\end{array}$ & $\begin{array}{c}\text { PFo } \\
\text { (mois) }\end{array}$ & $\begin{array}{c}\text { PFop } \\
(\%)\end{array}$ \\
\hline \multirow[b]{2}{*}{ Enshi } & Test de Pettitt & 1968 & 1968 & - & - & - & - & 1968 & - & - \\
\hline & Test de Hubert & 1968 & 1960 & - & 1979 & - & - & 1984 & 1968 & 1968 \\
\hline \multirow[b]{2}{*}{ Buakuc } & Test de Pettitt & 1968 & 1968 & - & - & - & - & - & - & - \\
\hline & Test de Hubert & 1968 & - & - & - & - & - & - & - & - \\
\hline
\end{tabular}

Tableau 9. Stationnarité des indices de pluies mensuels

Les résultats montrent que l'hypothèse d'une rupture est acceptée en 1968 pour deux (2) indices (PXMA et PA) à Buakuc et trois (3) indices (PXMA, $\mathrm{PA}$ et $\mathrm{PI}_{\mathrm{p}}$ ) à Enshi par le test de Pettitt. Par ailleurs, à cette dernière station le test de Hubert détecte une rupture en 1968 dans les indices PA, PFo et PFop puis une autre en 1979 avec l'indice PFap.

\section{Discussion}

L'analyse de la variabilité de la pluie extrême entre 1941 à 2000 sur le bassin versant de la Bia montre une abondance de la pluviométrie au sud du 
bassin (Adiaké et Enshi) induisant une décroissance pluviométrique du sud au nord. Une observation similaire sur le même bassin a été faite par Meledje (2016). Selon Eldin (1971), l'abondance des précipitations dans la zone sud du bassin est favorisée par une disposition plus perpendiculaire des secteurs Est et Ouest du littoral aux vents de mousson. Par ailleurs, la décroissance de la pluviométrie du sud au nord pourrait s'expliquer par l'effet de continentalisation, du fait de l'appauvrissement en eau de la masse d'air porteuse de précipitation au fur et à mesure de son avancée dans le nord du bassin de la Bia à Agnibilékro (Brou \& Dieulin, 2008).

La tendance linéaire de ces indices de pluie révèle que la baisse générale de la pluie extrême observée sur le bassin versant de la Bia de 1941 à 2000 n'a pas affecté le bassin versant de la même manière. En effet, les stations d'Adiaké en Côte d'Ivoire et de Enshi au Ghana restent les plus affectées avec une baisse très significative d'environ 0,48\% par décennie. Cependant, la tendance négative reste peu significative pour la majorité des indices étudiés aux stations d'Ayamé, de Bianouan, d'Agnibilékro et de Buakuc. Ces résultats montrent que de façon globale, la pluie extrême sur le bassin de la Bia n'a pas connu de changement significatif dans son évolution temporelle au cours de la période 1941-2000. Des observations similaires ont été faites en Côte d'Ivoire par Soro et al. (2013) avec les données de pluie extrêmes de courte durée, en Mauritanie par Ozer et al. (2014), sur le bassin de Chérif en Algérie par Taibi et al. (2015) et au Burkina par Hangnoh et al. (2015). Elles confirment ainsi les conclusions sur le rapport du groupe d'experts intergouvernemental quant à l'évolution du climat à l'échelle globale, à savoir des précipitations extrêmes inchangées dans un contexte de baisse globale (IPCC, 2013).

La stationnarité des indices de pluie étudiés révèle que les jours de pluie très extrêmes, de même que les indices de pluie mensuelle à Buakuc restent presque stationnaires. Ce qui justifie les tendances non significatives observées par régression linéaire (Brou et al., 1998). Cependant, l'occurrence des ruptures dans les indices non stationnaires s'observent majoritairement en 1968 pour les indices de pluie mensuelle. Cette date coïncide bien avec celle déterminée dans la plupart des études en Afrique de l'ouest et centrale (Conway et al., 2009; Ouédraogo et al., 1998). Toutefois, dans les indices de pluie journalière, les dates de ruptures s'insèrent dans la majorité des cas dans les ruptures dites précoces (Avant, 1960) et tardives (Après, 1980). À Agnibilékro, les doubles ruptures observées dans les indices non stationnaires s'insèrent dans la date des ruptures précoces (1948 et 1955) et tardives (1993 et 1995). La station de Bianouan présente également une rupture précoce (1953) et tardive (1981) dans les indices non stationnaires. Des observations concordantes en Afrique de l'ouest ont détecté des ruptures majoritairement en 1950 sur 33 stations (Hubert et al., 1989; 1998). En Côte d'Ivoire, sur le 
littoral, Fadiga (2012) a fait les mêmes observations à Sassandra en 1948. Les dates de ruptures observées aux stations d'Ayamé et d'Adiaké sont tardives. Ahoussi et al. (2013) ont révélé une rupture tardive (1982) à Adiaké avec des modules de pluie annuelle. Aussi, Fadiga et al. (2008) au Sud - Ouest de la Côte d'Ivoire ont détecté des ruptures tardives dans les modules de pluie annuelle de Grabo et de Tabou respectivement en 1981 et 1994. De plus, plusieurs études ont confirmé que les ruptures tardives seraient caractéristiques des régions côtières des pays bordiers du golfe de Guinée (Paturel et al., 1998; Brou et al., 2005). Cependant, des résultats récents (Noufe et al., 2012; Meledje et al., 2015) sur la zone étudiée révèle une rupture qui s'opère entre 1970 et 1972 s'insérant dans le contexte de l'évolution climatique observée de façon générale en Afrique de l'ouest et centrale. Cette différence entre les dates de ruptures observées peut se justifier par le faite que notre étude s'est portée sur des variables extrêmes issues de pluie journalière et mensuelle, et non des modules annuels. En effet, ce type de données peut apporter un complément quant à la variabilité de la pluviométrie (Servat et al., 1999; Assani et al., 1999). Ainsi, cette étude pourrait révéler que la rupture qui a eu lieu autour des années 1970 dans l'Est de la Côte d'Ivoire a affecté les extrêmes pluviométriques de la zone à partir des années 1980 révélant une rupture tardive.

\section{Conclusion Générale}

Cette étude a permis d'apprécier la variabilité de pluie extrême sur le bassin versant de la rivière Bia à partir de 1941 à 2000 en utilisant les indices de pluie.

L'analyse de la tendance de ces indices montre une baisse généralisée de la pluie extrême sur l'ensemble du bassin de 1941 à 2000 qui s'est révélée plus significative au sud du bassin (Adiaké et à Enshi).

En ce qui concerne la stationnarité, les dates de ruptures dans les indices de pluie journalière ne s'intègrent pas dans la période de rupture (1960 - 1970) du régime pluviométrique d'Afrique de l'ouest en général et non plus à la date moyenne (1972) dans notre zone d'étude. Ainsi, ces études pourraient révéler que la rupture intervenue autour des années 1970 dans l'Est de la Côte d'Ivoire a affecté les extrêmes pluviométriques de la zone à partir des années 1980 révélant une rupture tardive.

\section{References:}

1. Ahoussi, K. E., Koffi, Y. B., Kouassi, A. M., Soro, G., Soro, N. \& Biémi, J. (2013). Étude de la hydroclimatique et de ses conséquences sur les ressources en eau du Sud forestier et agricole de la Côte d'Ivoire : cas de la région d'Abidjan-Agboville. Int. J. Pure App. Biosci. Vol. 1, No. 6, pp. $30-50$. 
2. AssanI, A.A. (1999). Variabilité temporelle et persistance des épisodes secs en saison des pluies à Lubumbashi (Congo - Kinshasa). Note méthodologie, Sécheresse, Vol. 10, No. 1, pp. 45 - 53.

3. Bigot, S., Brou, Y. T., Bonnardot, V. \& Servat, E. (2002). Interannual stability of rainfall patterns in the Ivory Coast over the period 1950-1996. Publications de l'Association Internationale des Sciences Hydrologiques, pp. 507-514.

4. Beirot, P. (1965). Évolution des théories de la circulation atmosphérique générale. Annales de Géographie, Vol. 65, No. 348, pp. 81-97.

5. Boyer, J.F., Dieulin, C., Rouche, N., Cres, A., Servat, E., Paturel, J. E., \& Mahé, G. (2006). SIEREM an environmental information system for water resources. 5th World FRIEND Conference, La Havana - Cuba, November 2006 in Climate Variability and Change Hydrological Impacts IAHS Publ. 308, p19-25.

6. Brou, Y.T. \& Dieulin (2008). Atlas climatologique de Côte d'Ivoire. Hydrosciences Sierem. 20p.

7. Brou, Y.T., Servat, E. \& Paturel, J. E. (1998). Activités humaines et variabilité climatique : cas du sud forestier ivoirien. IAHS Publication, No. 252, pp. 365-373.

8. Brou, Y.T., Oszwald, Y., Bigot, S., \& Servat, E. (2005). Risques de déforestation dans le domaine permanent de l'État en Côte d'Ivoire : quel avenir pour ses derniers massifs forestiers ? TELA2004004, Revue de télédétection de l'Agence Universitaire de la Francophonie, pour le vol. 5, no 1-2-3, 2005, p 17-33.

9. Christensen, J., Carterand, T., \& Giorgi, F. (2002). Prudence employs new methods to assess European climate change. EOS, pp. 83 - 147.

10. Conway, D., Persechino, A., Ardoin, B. S., Hamandawana, H., Dieulin, C., \& Mahe, G. (2009). Rainfall and Water Resources Variability in Sub-Saharan Africa during the Twentieth Century. 19p.

11. Eldin (1971). Le climat. in : "Le milieu naturel de Côte d'Ivoire", Mémoire ORSTOM, Paris, No. 50, pp. 73 -108.

12. Fadika, V., Goula, B. T. A., Kouassi, F. W., Doumouya, I., Koffi, K., Kamagaté, B., Savané, I., \& Shrohourou, B. (2008). Variabilité interannuelle et saisonnière de l'écoulement de quatre cours d'eau de l'Ouest côtier de la Côte d'Ivoire (Tabou, Dodo, Néro et San pédro) Dans un contexte de baisse de la pluviométrie en Afrique de l'Ouest. European Journal of Scientific Research, Vol. 21, No. 3, pp. 406-418.

13. Fadika, V. (2012). Variabilité hydroclimatique et modélisation hydrologique de quelques bassins versants côtiers du Sud-ouest de la Côte d'Ivoire. Thèse unique doctorat, Université Abobo Adjamé. 
199 p.

14. Goula, A.B.T., Gneneyougo Soro, E., Kouassi, W., \& Srohourou, B. (2012). Tendances et ruptures au niveau des pluies journalières extrêmes en Côte d'Ivoire (Afrique de 1'Ouest). Journal-des Sciences Hydrologiques, Vol. 57, No. 6, pp. 1067-1080.

15. Hangnon, H., De Longueville, F., \& Ozer, P. (2015). Précipitations 'extrêmes' et inondations à Ouagadougou : quand le développement urbain est mal maîtrisé... XXVIIIe Colloque de l'Association Internationale de Climatologie, $6 \mathrm{p}$.

16. Hubert, P., Carbonel, J. P. \& Chaouche, A. (1989). Segmentation des séries hydrométéorologiques. Application à des séries de précipitations et de débits de l'Afrique de l'Ouest. Journal of Hydrology, 110, pp. 349-367.

17. Hubert, P., Servat, E., Paturel, J. E., Kouamé, B., Bendjoudi, H., Carbonel, J.P. \& Lubès Niel, H. (1998). La procédure de segmentation, dix ans après. IAHS Publication,. No. 252, pp. 267-273.

18. IPCC (2013). Climatic Change 2013: The Physical Science Basis. Contribution of Working Group I to the Fifth Assessment Report of the Intergorvernmental Panel on Climate Change. Cambridge University Press, Cambridge, United Kingdom and New York, USA, 1535 p.

19. Karimou Barké, M., Ambouta, K., Sarr, B., \& Tychon, B. (2015). Analyse des phénomènes climatiques extrêmes dans le sud-est du Niger. XXVIIIe Colloque de l'Association Internationale de Climatologie, $6 \mathrm{p}$.

20. Kouassi, A.M., Kouamé, K.F., Koffi, Y.B., Dje, K.B., Paturel, E.J., \& Oulare, S. (2010). Analyse de la variabilité climatique et de ses influences sur les régimes pluviométriques saisonniers en Afrique de l'Ouest : cas du bassin versant du N'zi (Bandama) en Côte d'Ivoire. Cybergeo, European Journal of Geography. 29p.

21. Lemaître, F. (2002). Recensement des tests de détection de tendances ou de ruptures l'analyse de stationnarité des régimes de crues en France. Rapport de fin d'études, Entpe, Cemagref-Lyon (France), 94p.

22. Lubès-Niel, H., Masson, J.M., Paturel, J.E. \& Servat, E. (1998). Variabilité climatique et statistique. Etude par simulation de la puissance et de la robustesse de quelques tests utilisés pour vérifier l'homogénéité de chroniques. Revue des Sciences de l'Eau, Vol. 11, No. 3 pp. 383 - 408.

23. Meledje, N.H.E (2016). Modélisation de la dynamique hydrologique et du flux des sédiments dans le lac du barrage hydroélectrique d'Ayamé 1. Thèse unique de doctorat, UFR-Sciences et Gestion de l'Environnement, Université Nangui Abrogoua, 215p. 
24. Meledje, N.H., Kouassi, K.L., N'go, Y.N. \& Savane, I. (2015). Caractérisation des occurrences de sécheresse dans le bassin hydrologique de la Bia transfrontalier entre la Côte d'Ivoire et le Ghana : contribution des chaînes de Markov. Cah Agric, Vol. 24, No.83, 12p.

25. Noufé, D., Lidon, B., Mahé, G., Servat, E., Brou, T., \& Koli Bi, Z. (2012). Variabilité climatique et production de maïs en culture pluviale dans l'Est ivoirien. Journal des Sciences Hydrologiques, vol. 56, No. 1 , pp. 52 - 67.

26. Ouedraogo, M., Lubès-H., Masson, Servat, E., \& Paturel, J.E. (1998). Caractérisation d'une modification éventuelle de la relation pluie-débit aux alentours de 1970 en Côte d'Ivoire Water ressources Variability in Africa during the XXth century. IAHS Publication, No. 2521, pp. 315-321.

27. Ozer, P., Hountondji, Y. C., Gassani, J., Djaby, B., \& De Longueville, F. (2014). Évolution récente des extrêmes pluviométriques en Mauritanie (1933-2010). XXVIIe Colloque de l'Association Internationale de Climatologie, $7 \mathrm{p}$.

28. Paturel, J.E., Servat, E. \& Delattre, M.O. (1998). Analyse de séries pluviométriques de longue durée en Afrique de l'Ouest et centrale non sahélienne dans un contexte de variabilité climatique », Journal des Sciences Hydrologiques, vol. 43, No.3, pp. 937- 945.

29. Pettitt, A.N. (1979). A non-parametric approach to the change-point problem. Applied Statistics 28 (2), pp. 126-135.

30. Reizer, C. (1967). Aménagement piscicole du lac artificiel d'Ayamé. CTFT, 108p.

31. Servat, E., Paturel, J.E., Kouamé, B., Travaglio, M., Ouédraogo, M., Boyer, J. F., Lubès-Niel, H., Fritsch, J.M., Masson, J.M., \& Marieu, B. (1998). Identification, caractérisation et conséquences d'une variabilité hydrologique en Afrique de l'Ouest et centrale » IAHS Publication, vol. 252, pp. 323 - 337.

32. Servat, E., Paturel, J.E., Lubès-Niel, H., Kouamé, B., Masson, J.M., Travaglio, M. \& Marieu, B. (1999). De différents aspects de la variabilité de la pluviométrie en Afrique de l'ouest et centrale non sahélienne ", Revue des Sciences de l'Eau, vol. 12, No.2, pp. 363-387.

33. Soro, G. E., Goula, B. T. A., Kouassi, F. W. \& Srohourou, B. (2013). Évolution des intensités maximales annuelles des pluies horaires en Côte d'Ivoire. Agronomie Africaine, 22 (1) : 33 - 44.

34. Taibi, S., Meddi, M. \& Mahé, G. (2015). Evolution des pluies extrêmes dans le bassin du Chéliff (Algérie) au cours des 40 dernières années 1971-2010. IAHS Publication, 369, pp. 175 - 180. 